\title{
Impairment of ultrastructure and cytoskeleton during progression of cardiac hypertrophy to heart failure
}

\author{
Anasuya Gupta ${ }^{1}$, Sudhiranjan Gupta ${ }^{1}$, David Young ${ }^{1}$, Biswajit Das ${ }^{1}$, James McMahon² and Subha Sen ${ }^{1}$
}

Studies at the morphological and molecular level have found that transgenic ( $\mathrm{Tg}$ ) mice that overexpress myotrophin in the heart develop hypertrophy at the early age of 4 weeks; this condition worsens to heart failure (HF) at approximately 36 weeks. However, how the sustained effects of alteration in cytoarchitecture of the contractile machinery lead to malfunction of the normal heart remains unclear. Our data have shown that at 4 weeks, the cytoarchitecture observed in left ventricular (LV) tissue samples of Tg mice is similar to that of wild-type (WT) mice. However, as the disease progresses, cardiomyocytes show deterioration in some mitochondrial as well as myofibril features, evidenced by swelling of mitochondria, misalignment of myofibril structure, and blurring as well as breakage of Z-lines. At 36 weeks of age, Tg mice (the group in transition from hypertrophy to HF) show significant degenerative changes in cardiomyocytes, including swelling of mitochondria, disruption of the nuclear membrane, and absence of myofibril structure. Besides these, formation of myelin bodies was also observed, a feature typically found in human hearts with HF. Changes in Z-line architecture were further confirmed by alteration in the gene expression profile of desmin and tubulin, the two main cytoskeletal proteins. We thus conclude that $\mathrm{Tg}$ mice overexpressing myotrophin show no visible changes in the initiation phase ( 4 weeks); however, as the disease progresses, alterations in the cytoskeleton are found during the transition phase from hypertrophy to HF (36 weeks onward). Our data suggest that treatment for prevention/reversal of hypertrophy should start at the early stage of hypertrophy to prevent its transition to HF.

Laboratory Investigation (2010) 90, 520-530; doi:10.1038/labinvest.2010.43; published online 15 February 2010

KEYWORDS: cardiomyopathy; desmin; myocytes; myotrophin; transgenic mice; tubulin

Heart failure (HF), the final common outcome of most primary cardiovascular diseases, is one of the most frequent causes of death worldwide. ${ }^{1}$ The potential causes of HF are diverse but often related to hypertrophy of cardiomyocytes and fibrosis., ${ }^{2,3}$ Among many such possible causes, left ventricular (LV) hypertrophy is a significant risk factor for cardiovascular diseases and $\mathrm{HF}^{4}$ Several factors might contribute to the increased susceptibility of hypertrophied myocardium, and among them, structural abnormalities may have significant deleterious effects. However, whether the injury is reversible and the cells ultimately recover or whether membrane disruptions occur, resulting in irreversible injury and death of the cell, depends on the intensity of these factors. ${ }^{5}$ In human hearts, the structural basis of the progression from well-compensated hypertrophy to HF is still largely unknown, and the correlation between morphological alterations and molecular changes during the different phases of the transition to HF has not yet been fully described. ${ }^{6}$
Studies from ultrastructural changes in patients with endstage dilated cardiomyopathy (DCM) have shown an increase in fibrosis, degeneration of hypertrophied myocardial cells, and alterations of the cytoskeleton. ${ }^{7}$ End-stage human $\mathrm{HF}$ is characterized by fibrosis as well as impairment of myocyte degeneration, ${ }^{7-9}$ apoptotic cell death, ${ }^{9}$ and HF markers such as myeloid structures. ${ }^{10-12}$

In recent years, many cardiomyopathies have been linked to mutations in either sarcomeric or cytoskeletal proteins, resulting in cardiovascular diseases, and several mouse models have been generated to study the structural and functional changes and elucidate the pathophysiology of the disease. In the cardiac hypertrophic mouse model involving Smad4, electron microscopic observations revealed abnormal mitochondria and disruption of Z-lines. ${ }^{13}$ Transgenic (Tg) mice with cardiac-restricted overexpression of tumor necrosis factor showed loss of sarcomeric registration and myofibril disarray as the animals aged. ${ }^{14}$

${ }^{1}$ Department of Molecular Cardiology, Lerner Research Institute, Cleveland Clinic, Cleveland, OH, USA and ${ }^{2}$ Department of Anatomic Pathology, Pathology and Laboratory Medicine Institute, Cleveland Clinic, Cleveland, OH, USA

Correspondence: Dr S Sen, PhD, DSC, Department of Molecular Cardiology, NB 50, Cleveland Clinic, 9500 Euclid Avenue, Cleveland, OH 44195, USA.

E-mail: sens@ccf.org

Received 26 May 2009; revised 16 September 2009; accepted 20 October 2009 
However, a comprehensive chronological study of structural correlates of HF-starting from the initiation of disease, during its progression, and continuing through the transition from hypertrophy to HF-is still wanting. In addition, a still unanswered question is whether molecular changes initiate the process of cardiac hypertrophy at an early stage and drive the myocardium to abnormal cytoarchitecture leading to HF, or the reverse. Moreover, as the heart is a complex organ and HF due to hypertrophy is a dynamic and intricate process, it is important to determine at which stage the changes in cytoarchitecture start to occur. It is also important to know whether changes in cytoarchitecture that contribute to HF arise from a particular area of the myocardium and then extend/spread to other parts as the severity increases or not.

We generated a Tg mouse model overexpressing myotrophin. ${ }^{15}$ At a young age, these mice develop cardiac hypertrophy that eventually leads to HF (approximately 36 weeks of age). Using this model, we documented the alteration of gene clusters that participate during the identification of hypertrophy and during the transition from hypertrophy to HF. Our Tg mouse model of hypertrophy is unique, because the disease course in these mice closely parallels that of human end-stage HF in both molecular (gene expression of hypertrophy marker genes, proto-oncogenes, cytokines, and growth factors) and physiological parameters (pleural effusion and lethargy). This model thus provides us the opportunity to study disease progression in vivo to dissect out the role of myotrophin-induced signaling pathways from the initiation of hypertrophy through its progression to HF. The uniqueness of this Tg model is that the structural remodeling of the myocardium as an effect of myotrophin-induced hypertrophy throughout the entire disease process can be studied in a sequential manner. This type of study is not possible with human heart samples. It is noteworthy that cardiac hypertrophy as a consequence of myotrophin overexpression has been found in this model as early as at 4 weeks of age and persists thereafter, resulting finally in HF at 36 weeks. Besides molecular changes, light-microscopic studies have also revealed some significant structural changes at the HF stage, results that indeed corroborate the idea that the myocardium also undergoes structural remodeling at the cellular level.

Cytoskeletal changes in myocytes have been documented during cardiac hypertrophy. ${ }^{16}$ An enhanced polymerization of tubulin and microtubule formation has been shown to correlate with an increase in cytoskeletal stiffness. Therefore, as a marker for cytoskeletal changes, we have quantified the changes in tubulin and desmin (both at the mRNA and protein level) during progression of cardiac hypertrophy to HF in the Tg mouse model.

The purposes of this study are to define (1) the mode of cytoarchitectural alteration at initiation to progression and transition of hypertrophy to HF and (2) the changes in cytoskeleton in parallel to ultrastructural changes during progression of hypertrophy to HF.

\section{MATERIALS AND METHODS Experimental Design \\ Animal model used}

To study the ultrastructural changes during the progression of cardiac hypertrophy in the LV, we have selected three different age groups of the Tg mouse model, generated in our laboratory, which develops hypertrophy early and progresses to $\mathrm{HF}$ as a result of overexpression of myotrophin in the heart. The Tg groups were compared with age-matched wildtype (WT) mice.

\section{Generation of $\mathrm{Tg}$ mice}

An $\alpha$-MHC-myotrophin transgene was constructed using a recombinant myotrophin (myo) in a pcDNA3 myo-vector, as described previously. ${ }^{15}$ Pronuclear injection was performed at the University of Cincinnati's transgenic facility, using standard techniques. This study was conducted with Tg mice from line 15.

All experiments performed were approved by the institutional animal care and use committee, according to internal policies and guidelines of the National Institutes of Health for the humane care and use of animals in research.

\section{Experimental groups}

$\mathrm{Tg}$ and WT animals $(n=18)$ were divided into three different groups based on the stage of the disease: initiation (group I), consisting of 4- to 6-week-old animals; progression (group II), consisting of 16- to 24-week-old animals; and transition from hypertrophy to HF (group III), consisting of animals of age 34-36 weeks. Three Tg animals from each group were studied, each along with their WT counterparts.

\section{Parameters chosen}

This study was focused on alteration in cardiomyocytes with particular reference to the sub-cellular organelles (mitochondria and nucleus) and also myofibril structure in general.

\section{Sampling procedure and imaging}

To have a random, unbiased, and global view of the samples studied, the experimental design was set up as follows. From each animal's LV (myocardial wall), three longitudinal pieces were taken from the front (subepicardial), middle (mid wall), and back (subendocardial) portions, each of which were then subdivided into a top and a bottom block. Many small pieces of tissue were prepared from each of these blocks, and finally one sample was selected at random for embedding.

The direction of sectioning was longitudinal for each case. At least 15 ultrathin sections were cut from each of the blocks and placed on copper grids.

Finally, one grid was randomly selected and each of the grids was studied under the electron microscope at four corners and at the center. Thus, for each heart (LV), a total of 30 possible locations were used for evaluation (Figure 1). 

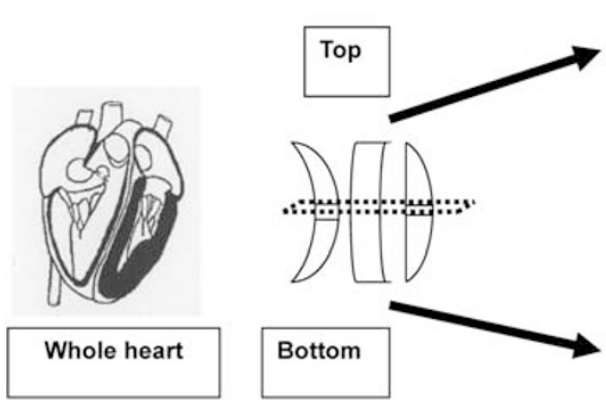

Front

Middle

Back

Figure 1 Schematic diagram of sampling procedure.

The micrographs taken from each zone were analyzed on a computer using the software Image Pro Plus (Version 6.0; Media Cybernetics, Bethesda, MD, USA) and Capture (Version 6.0).

\section{Morphometry/semiquantitative scoring}

To evaluate the degree of ultrastructural damage, a semiquantitative scoring system was used for cardiomyocytes focusing on subcellular structures such as mitochondria, nucleus, and myofibril arrangement (Table 1). Test fields were selected at a primary magnification of $3000 \times$ by systematic uniform random sampling. Areas of ultrastructural damage were assessed according to a four-point scale for all of these parameters. ${ }^{5}$ The EM pictures were read by blinded readers, who were not aware of the groups or genotypes of the mice.

\section{Tissue processing for electron microscopy}

Both WT and Tg mice were euthanized by carbon dioxide, then $0.1 \mathrm{ml} 10 \%$ potassium chloride was injected to the $\mathrm{LV}$ to stop the heart at diastole. The hearts were removed, washed in phosphate-buffered saline (PBS), and kept in cardioplegic solution ${ }^{17}$ for $1 \mathrm{~min}$, followed by incubation in $2.5 \%$ glutaraldehyde and $4 \%$ formaldehyde $\left(4{ }^{\circ} \mathrm{C}\right.$ overnight). The LVs were then separated and cut into pieces. The pieces were post fixed in 1\% osmium tetraoxide and embedded using an Epon 812 kit. Ultrathin $(85 \mathrm{~nm})$ sections were cut and stained with uranyl acetate and lead citrate and examined under a Philips CM12 transmission electron microscope at a voltage of $60 \mathrm{kV}$.

\section{Data collection and statistical analysis}

All data collected from the WT and Tg animals of different groups were coded, recorded, and analyzed. Mann-Whitney statistical analyses were used for comparing differences among groups. Data were expressed as the mean value \pm s.e.m., and $P<0.05$ was accepted as statistically significant. Statistical analyses showed that the values obtained from different zones were not significantly different, and the changes were homogeneously distributed throughout the
Table 1 Scoring parameters for assessing the ultrastructural alteration in hearts

\begin{tabular}{lc}
\hline Category & Type \\
\hline Mitochondria & 1 \\
Unchanged; electron-dense matrix with granules and & \\
densely packed cristae & 2 \\
Matrix locally electron lucent; loss of granules & 3 \\
Swelling and/or fragmentation of cristae & 4 \\
Presence of concentric cristae &
\end{tabular}

Myofibril structure and Z-line

Parallel with all the bands 'Z-lines' visible 1

Wavy Z-line

Blurring, breakage, thickening of Z-line 3

No myofibril structure 4

Cytoplasm and nucleus

Round/oval shape, intact nucleolus, homogeneous chromatin, and continuous basal lamina

Change in shape

Convoluted nuclear membrane

3

Rupture of cell membrane and/or karyolysis

tissue samples. Therefore, only mean values from all the samples were considered.

For the three groups (initiation, progression, and transition), total counts were as shown: mitochondria, 3600 (WT, 1800 ; Tg, 1800); nuclei, 110 (WT, 54; Tg, 56); and myofibrils, 587 (WT, 310; Tg, 277).

\section{Distribution per Zone of the Changed Organelles}

To assess whether there was any 'zone-wise' bias in the intensity of damage, each heart sample was critically examined. 
LVs of the heart samples from transition-phase animals of each type (WT and Tg) were divided into six parts, which were randomly chosen to assure a global representation. The data counted from each test field were critically analyzed.

\section{Western Blot Analysis}

Hearts of 4-, 16-, and 36-week-old WT and Tg mice were aseptically taken out and washed with cold PBS to remove the blood. Hearts were then minced with a sterile blade and lysed on ice in a buffer containing $10 \mathrm{mM}$ Tris- $\mathrm{Cl}(\mathrm{pH} 7.5)$, $150 \mathrm{mM}$ sodium chloride, $5 \mathrm{mM}$ magnesium chloride, $1 \mathrm{mM}$ ethylenediaminetetraacetic acid, $10 \mathrm{mM}$ sodium pyrophosphate, $10 \%$ glycerol, $50 \mathrm{mM}$ sodium fluoride, $100 \mathrm{M}$ sodium orthovanadate, $10 \mathrm{nM}$ okadaic acid, $0.5 \mathrm{mM}$ dithiothreitol, $1 \mathrm{mM}$ phenylmethylsulfonyl fluoride, $1 \%$ Triton-X-100, $1 \mu \mathrm{g} /$ $\mathrm{ml}$ leupeptin, $10 \mu \mathrm{g} / \mathrm{ml}$ aprotinin, and $1 \mu \mathrm{g} / \mathrm{ml}$ pepstatin. The lysates were centrifuged at $14000 \times g$ for $20 \mathrm{~min}$ at $4{ }^{\circ} \mathrm{C}$. The total protein concentration was measured by the Bradford method. Samples containing $50 \mu \mathrm{g}$ of protein were separated on $10 \%$ sodium dodecyl sulfate-polyacrylamide gels and were electrophoretically transferred onto polyvinylidene fluoride membrane using a wet transfer apparatus (Bio-Rad Laboratories, Hercules, CA, USA). Membranes were incubated in a blocking buffer containing 5\% nonfat dry milk (Bio-Rad) in Tris-buffered saline with $0.1 \%$ Tween-20. Membranes were probed with desmin and tubulin antibodies (Sigma), overnight at $4{ }^{\circ} \mathrm{C}$ (all at 1:1000 dilution), washed three times in Tris-buffered saline with $0.1 \%$ Tween- 20 and then detected using a horseradish peroxidase-conjugated secondary antibody and enhanced chemiluminescence (NEN Life Sciences, Boston, MA, USA). Glyceraldehyde 3-phosphate dehydrogenase (GAPDH) was used as an internal protein loading control.

\section{RNA Extraction and Semiquantitative RT-PCR}

Hearts from WT and Tg mice of 4, 16, and 36 weeks of age were aseptically taken out and washed with cold PBS to remove the blood. Hearts were then minced with a sterile blade and total RNA was isolated from the heart of WT and $\mathrm{Tg}$ mice according to the protocol of Chomczynski and Sacchi. ${ }^{18}$ RNA was re-suspended in diethylpyrocarbonate water and quantitated by optical density at $260 \mathrm{~nm}$. The total RNA was treated with DNase I to remove genomic DNA contamination. By running an aliquot of the RNA sample on a denaturing agarose gel, we assessed the quality of the RNA. The first-strand cDNA reaction was performed with M-MLV (Moloney murine leukemia virus) reverse transcriptase (Invitrogen, Carlsbad, CA, USA) and oligo-dT primers from $1 \mu \mathrm{g}$ of total RNA. PCR amplification was performed with the same amount of the first-strand cDNA using Taq DNA polymerase (Invitrogen). The PCR protocol consisted of 30 cycles each of $94{ }^{\circ} \mathrm{C}$ for $30 \mathrm{~s}, 55^{\circ} \mathrm{C}$ for $1 \mathrm{~min}$, and $72{ }^{\circ} \mathrm{C}$ for $30 \mathrm{~s}$, followed by an extension at $72{ }^{\circ} \mathrm{C}$ for $5 \mathrm{~min}$; GAPDH was used as a loading control for the amount of input RNA. Mouse desmin and tubulin PCR primers were designed based on the GenBank nucleotide sequences for accession numbers L22550 and X04663, respectively (National Center for Biotechnology Information, Bethesda, MD, USA). The primer sequences of desmin and tubulin are given below:

\section{Desmin sense: $5^{\prime}$-TCTACGAGGAGGAGATGC-3' Antisense: $5^{\prime}$-ATCGTTGTTCTTATTGGCTGCC-3' Tubulin sense: $5^{\prime}$-CACAGGTGGCAAGTATGTCC- $3^{\prime}$ Antisense: 5'-GGTCTCATCCGTGTTCTAACC-3'}

The PCR product sizes for desmin and tubulin were $475 \mathrm{bp}$ and $435 \mathrm{bp}$, respectively. The amplified products were loaded and separated on $1 \%$ agarose gel and stained with ethidium bromide.

\section{RESULTS}

\section{Changes in Mitochondria}

Initiation phase (group I: 4-6 weeks of age)

No structural abnormalities were observed in $\mathrm{Tg}$ mice at the initiation phase (group I) when compared with WT mice. The mitochondria are well preserved, possessing dense matrices filled with homogeneous matrix granules, intact double membrane, and tightly packed cristae (Figure $2 \mathrm{a}$ and $\mathrm{b}$ ).

\section{Progression phase (group II: 16-24 weeks of age)}

WT animals in the progression phase (group II) also showed no conformational change in mitochondria (Figure 2c). In $\mathrm{Tg}$ animals, however, a variety of changes were observed, depending on the severity of damage. The most obvious alterations were loss of matrix granules and the appearance of electron-lucent areas $(P<0.05$; Figure $2 \mathrm{~d})$. Mitochondrial swelling $(P<0.05)$ has also started to occur (Figure $2 \mathrm{e})$.

\section{Transition phase from hypertrophy to HF (group III: 34 weeks onward)}

The transition-phase WT mice revealed more or less normal features (Figure 2f), with a few exceptions. It is important to note that, before 36 weeks, no alteration in mitochondrial ultrastructure was ever observed in WT animals, but not significant when compared with results from the preceding age group. In Tg animals of group III, loss of matrix granules, which started earlier in the younger/previous group, showed a significant $(P<0.05)$ increase in the transition phase (Figure $2 \mathrm{~g})$. Mitochondria were more swollen $(P=0.002$; Figure $2 \mathrm{~h}$ ). Changes, such as the appearance of concentric cristae, were found to be significant in the Tg animals of group III $(P=0.0005$; Figure $2 \mathrm{i})$.

\section{Changes in Nucleus}

\section{Initiation phase (group I: 4-6 weeks of age)}

Nuclear morphology did not differ much from normal features in both WT and Tg mice at the initiation phase. All nuclei were more or less round to elliptical with a homogeneous chromatin network, intact nucleoli, and thin, continuous basal lamina (Figure $3 \mathrm{a}$ and $\mathrm{b}$ ). 

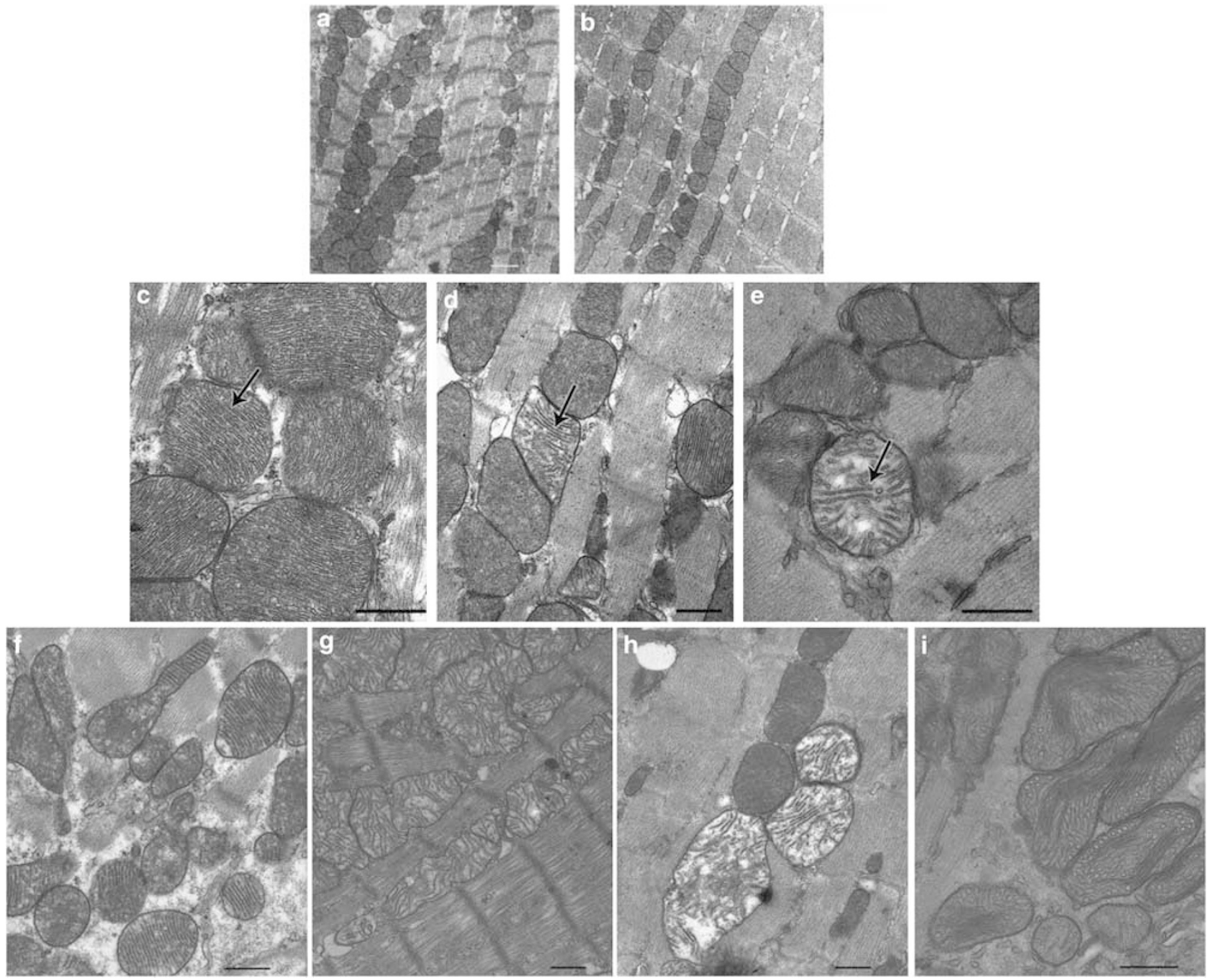

Figure 2 ( $\mathbf{a}$ and $\mathbf{b}$ ) Electron photomicrographs of the cardiomyocytes of WT (a) and $\mathrm{Tg}(\mathbf{b})$ animals (initiation phase) showing well-preserved mitochondrial (Mt) features. No apparent changes were observed in Tg animals when compared with WT mice (bar $=1 \mu \mathrm{m})$. (c-e) Electron photomicrographs of the cardiomyocytes of WT (c) and Tg (d and e) animals (progression phase) showing mitochondrial changes. WT mice show normal features such as organized cristae and intact membrane structure, whereas Tg mice show changes such as electron-lucent areas (d) and swelling (e) $(b a r=0.5 \mu \mathrm{m})$. (f-i) Electron photomicrographs of the cardiomyocytes of WT (f) and Tg (g-i) animals (transition phase) showing mitochondrial changes in most severely damaged areas. WT mice show normal mitochondrial contour/ structural integrity (though not typically linearly arranged), whereas Tg mice show loss of matrix (g), and severely dilated (h) and paraconcentric cristae (i) $(\mathrm{bar}=0.5 \mu \mathrm{m})$.

\section{Progression phase (group II: 16-24 weeks of age)}

Nuclei from WT mice in the progression phase did not show any significant changes (Figure $3 \mathrm{c}$ ). In Tg animals of group III, the most prevalent alteration was deformation in shape of the nucleus. Nuclei sometimes appeared somewhat elongated, crescent shaped, and often bizarre $(P<0.001$; Figure $3 \mathrm{~d})$. Sometimes the nuclear membranes were found convoluted (Figure 3e). Some myocytes of transgenic tissue showed ruptured nuclei along with rupture of nucleoli (data not shown); however, the number of occurrences was not significant.

\section{Transition phase from hypertrophy to HF (group III: 36 weeks onward)}

Tissue of WT mice in the transition phase revealed no divergence from normal nuclear contour (Figure 3f). In $\mathrm{Tg}$ mice, considerable structural alterations were quite evident at the onset of the transition phase. Distortion of nuclear shape was persistent in this group (Figure 3g) and severity of the disease was manifested as more convolution of nuclear membrane (Figure 3h; $P<0.0002$ ). Among the Tg animals of group III, rupture of nuclear membrane and karyolysis were even more frequently observed (Figure $3 i ; P<0.0001$ ) when compared with their WT counterparts and mice in group II.

\section{Changes in Myofibril Structure/Z-Line} Initiation phase (group I: 4-6 weeks of age)

The changes occurring in myofibril and Z-line structure reflect the contractile properties of the heart. In general, both WT (Figure 4a) and Tg mice (Figure 4b) in the initiation phase (group I) showed normal ultrastructural features of 


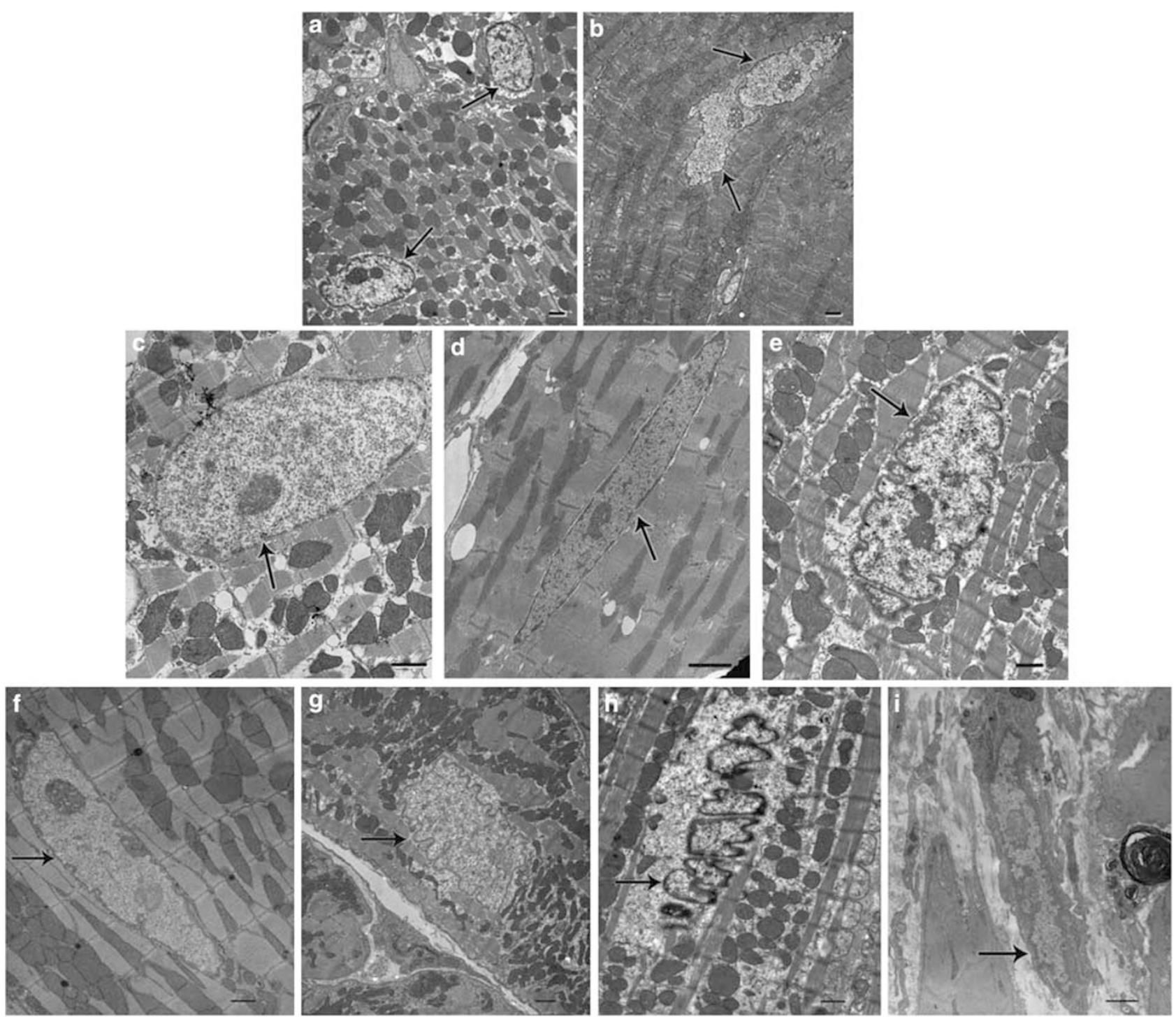

Figure 3 (a and $\mathbf{b}$ ) Electron photomicrographs of the cardiomyocytes of WT (a) and Tg (b) animals (initiation phase) showing nuclear changes. Tissues from both WT and Tg mice show well-preserved nuclear (N) structure (bar $=1 \mu \mathrm{m}$ ). (c-e) Electron photomicrographs of the cardiomyocytes of WT (c) and Tg (d and e) animals (progression phase) showing altered nuclear features. Note the homogeneous chromatin material, prominent nucleolus (Nu), and intact basal lamina (BI) in WT tissue. Nucleus from Tg mice shows elongated morphology (d) with smooth nuclear envelope and intact nucleolus (Nu). These nuclei also show an irregularly folded nuclear envelope (e) in many cases (bar $=1 \mu \mathrm{m})$. (f-i) Electron photomicrographs of the cardiomyocytes of WT (f) and Tg (g-i) animals (transition phase) showing altered nuclear features. WT nucleus is well preserved. In Tg tissue, altered nuclear (N) structure shows elongated / bizarre morphology with smooth nuclear envelope (g). Nuclei in Tg tissue show convoluted nuclear envelope (h) with intranuclear tubule formation and abundant small mitochondria in severely affected areas. (i) Total disruption in nuclear structure is shown. The cell contains mostly unspecified cytoplasm (cyt) and almost no strands of myofibril electron-dense, concentric lamellar body or 'Myelin body' (Mb) is also observed to be present (bar $=1 \mu \mathrm{m}$ ).

myocardial cells. They had parallel arrays of myofibrils to the longitudinal cell axis with the mitochondria. A- and I-bands, and Z-lines in the I-bands, were clearly observed.

\section{Progression phase (group II: 16-24 weeks of age)}

WT animals showed no disruption in the composition or alignment of myofibril structures (Figure 4c). In the $\mathrm{Tg}$ animals, the parallel and linear arrangement of the Z-lines was found to be lost with progression of the disease, and the Z-lines appeared somewhat wavy in nature (Figure 4d), although the distance between two consecutive Z-lines was maintained. In some of the samples from $\mathrm{Tg}$ mice, ultrastructural analysis showed signs of sarcolysis. We could not detect any Z-lines (Figure 4e).

\section{Transition phase from hypertrophy to HF (group III: 36 weeks onward)}

In the transition phase, WT animals showed no significant distortion in structural organization of myofibrils (Figure 4f). In Tg animals, the wavy Z-lines were more prominent 

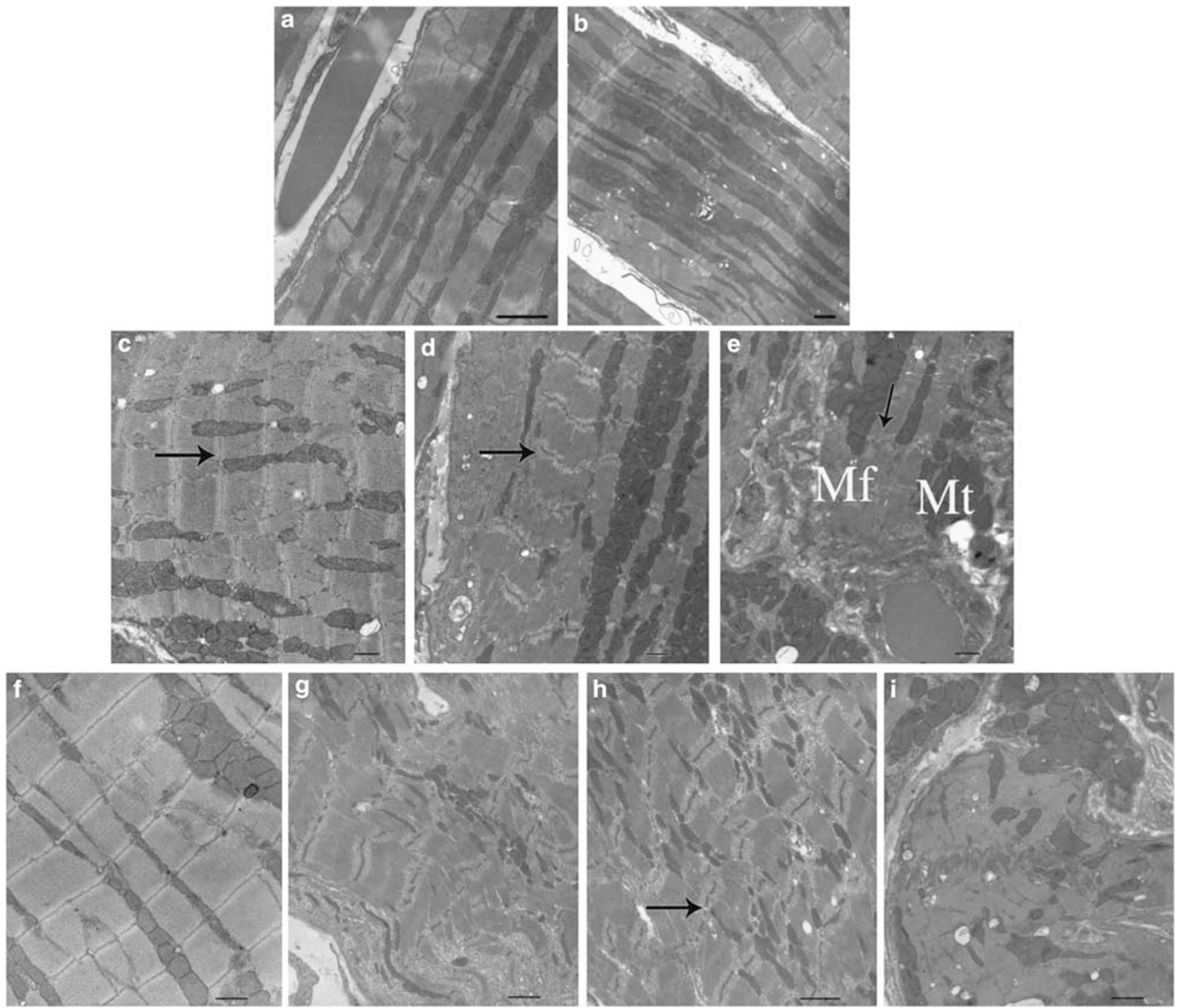

Figure 4 (a and $\mathbf{b}$ ) Electron photomicrographs of the cardiomyocytes of WT (a) and $\mathrm{Tg}(\mathbf{b})$ animals (initiation phase) showing linear myofibril arrangement in both the cases (bar $=1 \mu \mathrm{m}$ ). (c-e) Electron photomicrographs of the cardiomyocytes of WT (c) and $\mathrm{Tg}(\mathbf{d},-\mathbf{e})$ animals (progression phase) showing myofibril structural changes. WT shows equally distant straight Z-lines (arrow). Tg tissue shows disorganized Z-line following wavy contour (d). However, distances between Z-lines are maintained. e Area of severely affected myocyte is shown. Note the clusters of mitochondria (Mt) not maintaining linear orientation, few myofibrils (Mf) with distorted Z-lines (arrow), and myofibril-free cytoplasm (Bar $=1 \mu \mathrm{m}$ ). (f-i) Electron photomicrographs of the cardiomyocytes of WT (f) and $\operatorname{Tg}(\mathbf{g}, \mathbf{h}, \mathbf{i})$ animals (transition phase) showing altered myofibril structures. WT tissue is almost well preserved with linear Z-line structure. Tg tissue shows a variety of changes. The Z-lines are more wavy and not equally distant from one another (g). (h) Moderately distorted area of cardiomyocyte with blurring and breakage of Z-lines is shown. In severely affected zones, there is total loss of contractile material (i) showing large areas of cytoplasm, scattered arranged mitochondria (Mt), no Z-line, and fibrotic tissue (F) (bar $=1 \mu \mathrm{m})$.

along with a failure to maintain distance between two consecutive lines (Figure $4 \mathrm{~g}$ ). In the most severe cases, we observed partial blurring and breakage of Z-lines $(P<0.0001$; Figure $4 \mathrm{~h})$. As far as myofibril arrangement is concerned, in some cases, myocytes of the Tg animals with HF showed total disruption of the contractile system, and areas were found in which no myofibrils were observed (Figure 4i; $P<0.0001$ ). Besides their ultrastructural changes, accumulation of typical myelin bodies in the mitochondrial populations was discernable only in the group III Tg mice. In severely affected areas of the LV, different shapes of myelin bodies were found (Figure 5).

To monitor the relative transcriptional regulation of desmin and tubulin, we performed RT-PCR using the primer designed based on the GenBank sequences of mouse desmin and tubulin. The representative picture showed the profile for both desmin ( $475 \mathrm{bp}$ ) and tubulin ( $435 \mathrm{bp}$ ) at 4,16 , and 36 weeks of age with their age-matched WT counterparts. Our data showed that upregulation of tubulin starts at 16weeks of age in Tg mice when compared with their WT counterparts 


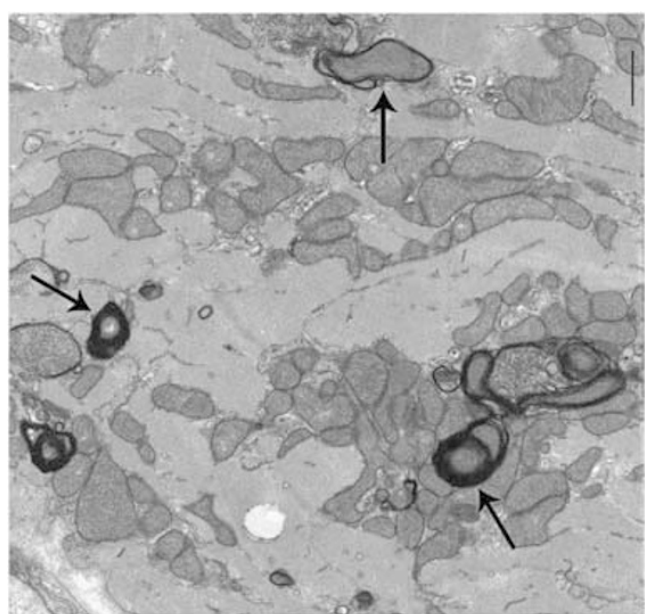

Figure 5 Electron photomicrograph of 'Myelin body' (arrow) in LV myocytes of $\mathrm{Tg}$ animal at transition from hypertrophy to heart failure (bar $=1 \mu \mathrm{m})$.

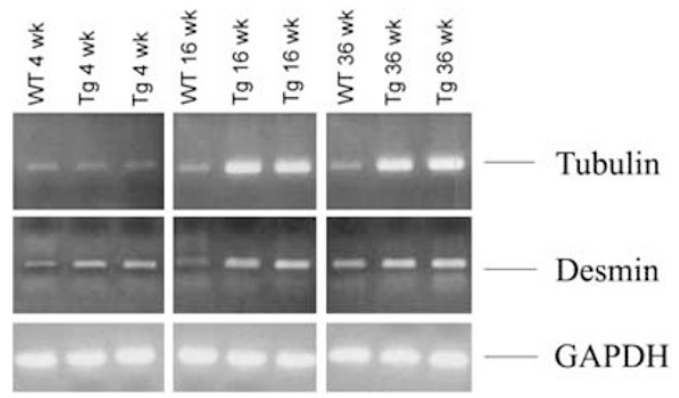

Figure 6 RT-PCR analysis of desmin and tubulin. Total RNA was extracted from WT and Tg mice hearts at 4, 16, and 36 weeks of age. Semiquantitative PCR was performed using first-strand CDNA from each of the WT and Tg hearts using their gene-specific primers as described in the Materials and Methods section. GAPDH was used as an internal RNA loading control. Results are presented in three separate experiments $(P<0.001$ compared with corresponding WT counterparts).

(3.1- and 3.2-fold, respectively, $P<0.001$ ) but not in 4 -weekold Tg mice. Similarly, we observed an upregulation of desmin starting at 4 weeks of age $(1.7$-fold, $P<0.001)$ and remained elevated by 35 weeks (2.2-fold and 2.4-fold, respectively, $P<0.001)$. We observed an upregulation of desmin and tubulin starting at 16 weeks of age in Tg mice but not in WT mice, and levels remained elevated in the Tg mice to 36 weeks of age (Figure 6).

To further relate their protein expression profile during progression of cardiac hypertrophy, we performed immunoblot analysis using specific antibodies. The results are summarized in Figure 7.

Our data showed that in Myo-Tg mice of all ages tested, cardiac desmin levels were significantly higher (2.4-, 2.8-, and 3.7 -fold, respectively, $P<0.001)$ than in the corresponding age-matched WT. Similarly, we found slight enhancement of tubulin expression level in Myo-Tg (1.29-, 1.32-, and 1.52 -fold, respectively, $n=3, P<0.001)$ compared with agematched WT.

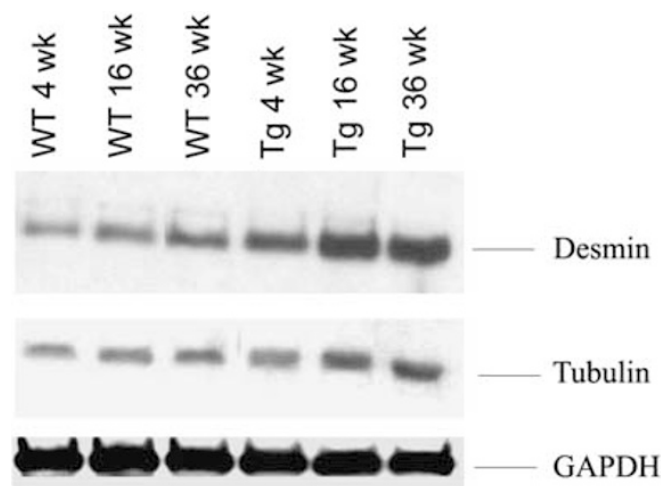

Figure 7 Western blot analysis of desmin and tubulin. Total cardiac lysate was prepared from WT and Tg mice hearts at 4, 16, and 36 weeks of age. Western blotting was performed using their specific antibodies described in the Materials and Methods section. Results are presented in three separate experiments $(P<0.001$ compared with WT mice).

\section{DISCUSSION}

The purpose of this study was to delineate the cytoarchitectural correlates of cardiac failure starting from initiation and progression through transition from hypertrophy to HF in a $\mathrm{Tg}$ mouse model overexpressing myotrophin. A careful analysis of electron microscopic studies showed that several types of subcellular alterations occurred in the LV of Tg mice when compared with their WT counterparts, with the advent of disease that reached extremity at the HF state. Tg animals of younger age did not show any detectable/significant changes in the cellular architecture, in spite of the fact that the process of hypertrophy has already begun, as evidenced by the molecular data on the same animal.

The experimental design for this study renders a random, unbiased, blinded representative sampling. Data show that there are significant and consistent changes in the Tg animals as the disease progresses. However, some changes were also observed among old WT mice, as noted below.

\section{Mitochondrial Changes}

Our data show that cardiac hypertrophy is associated with several types of changes in mitochondria, a vital organelle in the myocyte structure. However, no change was observed in the ultrastructure at the initiation phase, which may indicate that at that point the heart is not yet under a stressful condition of high oxygen demand. Loss of mitochondrial granules was among the early subcellular changes observed in $\mathrm{Tg}$ mice in the progression phase (group II; $P<0.05$ ). The native mitochondrial granules are known to disappear not only in the setting of hypoxia, but also in states of high oxygen stress. ${ }^{19}$ This finding explains the increasing oxygen demand of the cells in the present model as the disease progresses.

Another ultrastructural feature that was particularly prominent in this study was the swelling of mitochondria, which started as early as the progression phase (group II) in Tg mice. The Tg mice in HF showed the greatest number of swollen 
mitochondria. Interestingly, age-matched WT animals also showed some swollen mitochondria among the normal mitochondrial population, which was significant when compared with mice in the progression phase $(P<0.05)$. Studies have shown that mitochondria appear swollen when adenosine- $5^{\prime}$-triphosphate (ATP) content is lost, and openings appear suddenly of translucent spaces between cristae, leading to a decrease in oxidative phosphorylation. ${ }^{20}$ Hence, it is noteworthy that higher energy demand is a natural consequence of the aging process, which is manifested prema- turely in Tg animals as an effect of hypertrophic disease. Some researchers have shown a relationship between ATP, lactate changes, and mitochondrial swelling. If the changes were marked, the swelling was marked as well. Conversely, a lesser degree of change was associated with less swelling. ${ }^{4}$ However, as the disease progressed, the earlier change that was noted stopped progressing, and no further deterioration in mitochondria was observed among the HF group (group III) animals (Figure 8). The ratio of normal to swollen mitochondria rose with increasing severity of disease (Figure 8).

a Mitochondrial changes in Transgenic tissue $(n=1800)$
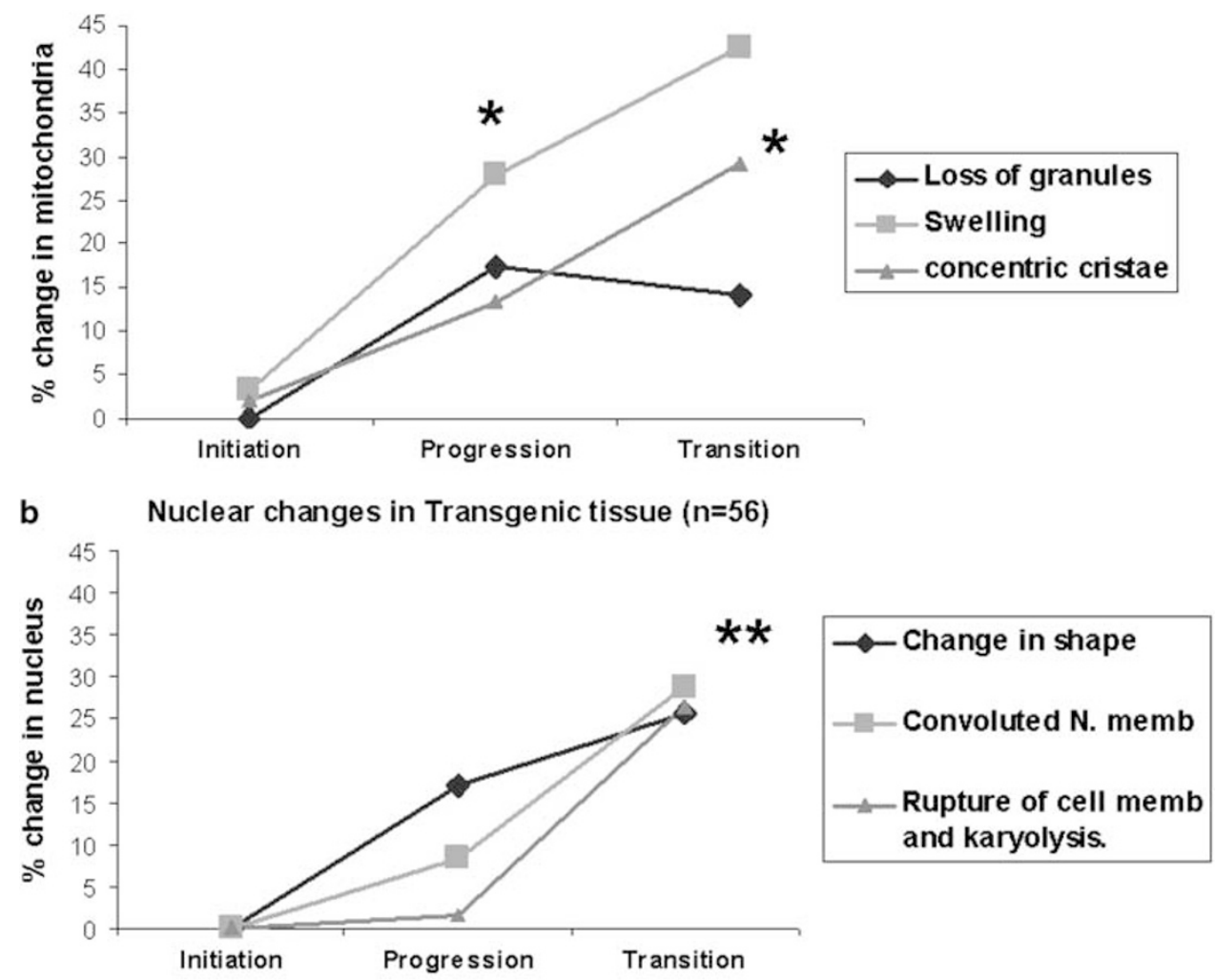

c Changes in myofibril structure in Transgenic tissue

$(n=277)$

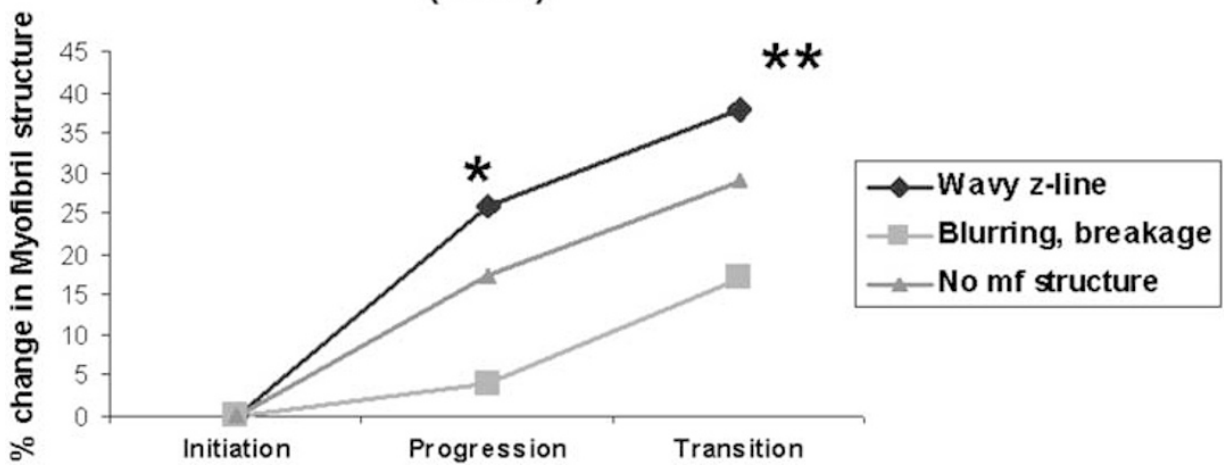

Figure 8 Graphical representation of types of changes observed in subcellular ultrastructure in Tg animals of different ages. Percentage of changes in (a) mitochondrial, (b) nuclear, and (c) myofibril structure during initiation, progression, and transition from hypertrophy to heart failure $(n=$ number of total structures, $P<0.05)$. Abbreviated names of different types of changes are provided. 


\section{Nuclear Changes}

Changes to the cell nucleus were also found to be fairly consistent in our study. Hypertrophic cardiomyocytes with mild-to-moderate degeneration showed deviations from the normal shape and an increase in convolutions of the nuclear membrane. It has been postulated that during ventricular hypertrophy, the length of the nucleus increases whereas its diameter remains normal. In addition, there is increased synthesis of nuclear membrane to accommodate the increased volume, which leads to increases in folding, convolutions, and deformation in shape. ${ }^{21}$ The appearance of convolutions in the nuclear membrane of $\mathrm{Tg}$ mice in the progression phase, as observed in our study, corroborates this idea.

The elongated and bizarre shapes observed in the nuclei of Tg mice (mild in progression-phase and severe in transitionphase animals) suggest that longitudinal and radial forces generated by ventricular chamber dilation may further exacerbate the effects of physical stress as the disease advances. Although the consequences of altered shape and size are yet to be defined, adverse effects on nuclear organization and function are likely. Structural changes in nuclear envelopes have been observed in cardiomyocytes in which the lamin $\mathrm{A} / \mathrm{C}$ gene is abnormal. ${ }^{22}$

In addition, the occurrence of altered nuclear morphology suggests that there may be defects in nuclear pore transport and/or transcription regulation. ${ }^{23}$ In this study, altered nuclear shape, size, and intranuclear organization, as well as dilation of the nuclear envelope shape (convolution), might cause or accompany changes in nuclear pore structure that would critically influence nuclear cytoplasmic transport and, hence, a range of nuclear functions.

\section{Myofibril Structural Changes}

Sarcomeric disorganization and Z-line abnormalities were found in myocytes from Tg mice, starting from the progression phase in this study. It is interesting to note that not all the sections from an individual mouse showed this misalignment. This discrepancy may have been because of a greater degree of variability in the local conditions under which the tissues are taken. However, as hypertrophic disease progresses, the misalignment becomes severe in Tg animals in the transition phase, no matter from which site the tissue was collected. Breakage and blurring of Z-lines may result from the non-uniform stretching of the myocytes, which are found to be present in the transition phase. The severity of tissue injury reaches its maximum level in $\mathrm{Tg}$ animals in the transition phase and is manifested by a total loss of myofibrils. Compensating for this loss of myofibrils, there is an increase in unspecified cytoplasm, fibrotic material, and interstitial collagen fiber. ${ }^{24}$ This other matter may be responsible for the stiffness of the ventricular wall observed at the end stage of dilated cardiomyopathy.

The Z-disc is involved in sarcomeric organization, force generation, and transmission and exerts an effect as an interface between contractile proteins, other cytoskeletal elements, and signaling molecules.

Desmin, the major intermediate filament in the sarcomere, is thought to be a major stress-bearing element there. It forms a ring-like band around myofibrils at the Z-line level with longitudinal bands running parallel to the myofibrils in the interfibrillar space and connecting mitochondria and desmosomes at the cardiac intercalated discs. ${ }^{25}$ In our Myo-Tg model, we observed an upregulation of desmin at both the transcriptional and translational level in compensated hypertrophy and further increased in decompensated hypertrophied hearts.

Tubulin exists as polymer-forming microtubules (a major component of the cytoskeleton in the myocyte) and has been found to increase in human and animal models of cardiac hypertrophy and HF. Enhanced tubulin polymerization and microtubule formation have been found to correlate with a concomitant increase in cytoskeletal stiffness. ${ }^{26,27}$

It is possible that the functional abnormalities associated with the cardiac hypertrophy in these $\mathrm{Tg}$ mice may be related to the hyperpolymerization of structural proteins such as tubulin and desmin. Specifically, densification of the microtubule and intermediate filament networks of the myocyte would likely impose a greater load on the myocyte and exert an effect to reduce the compliance of the cell and impede sarcomere motion and force production. Thus, increased tubulin and desmin protein levels in Myo-Tg mice may represent an important inciting mechanism for HF.

The most unique feature observed in this study is the random and homogeneous occurrence of myelin bodies, at different phases of formation, in the $\mathrm{Tg}$ mice in the transition phase. Myelin figures are indicative of mitochondrial damage ${ }^{10}$ when found in human HF samples, but it was not very clear at which point in time they occur during the progression of disease. Our extensive study in the hypertrophic murine model, starting from initiation and progression through transition from hypertrophy to HF, documented that these structures start to appear only at the end stage, and also, the formation of myelin body is a sequential phenomenon rather than a sudden mitochondrial alteration. However, an in-depth study on the formation of the said structure has yet to be conducted.

A zone-wise distribution of the altered organelles representing the severity of damage to the cardiomyocytes was critically scrutinized for the first time during the study, in which we used a thorough, random sampling method. It appears that there is no zone-wise bias with regard to the severity of damage, and it is more likely that the damage we found is homogeneous in distribution.

Our current study has shown that the complicated and comprehensive process of transition from hypertrophy to HF is associated with abnormal cardiac ultrastructure in the $\mathrm{LV}$ of Tg mice that overexpress myotrophin. The changes involve three main cell components, namely, mitochondria, nucleus, and myofibril structure. In conclusion, the effects of cardiac 
hypertrophy are observed at the molecular and biochemical as well as functional level, before there is any sign of structural abnormalities. Our findings also suggest that alterations in the cytoarchitecture of cardiomyocytes may contribute to further deterioration in the functioning of heart, but only at the point when the disease has already started; it appears that these structural anomalies are less likely to be involved in bringing about the initiation process of the disease. However, the extent of injury to the cells amplifies as the disease advances, reaching extremity at the transition phase from hypertrophy to HF. Hence, we conclude that the subcellular structural changes observed in this study serve to further promote the deterioration, already initiated by some other factors, possibly molecular changes. Moreover, the sampling procedures we used rendered a uniform, homogeneous, and representative study of the samples, which clearly indicates that the subcellular changes observed in this study are global and not focal.

\section{ACKNOWLEDGEMENTS}

We acknowledge Ms Yin Mei (Imaging Facility and Histology Core, Cleveland Clinic) for her expert technical assistance in tissue preparation for TEM, Mr Robert (Sam) Butler (Department of Quantitative Health Sciences, Lerner Research Institute, Cleveland Clinic) for assistance in statistical analyses, Mr Jim Lang (Photography Core, Lerner Research Institute, Cleveland Clinic) for his services, and the expert secretarial help of Michele Barnard of our department. This study was supported by a grant from the National Institutes of Health to Subha Sen (NIH R0-1 HL-47794).

\section{DISCLOSURE/CONFLICT OF INTEREST}

The authors declare no conflict of interest.

1. Braunwald $E$, Bristow MR. Congestive heart failure: fifty years of progress. Circulation 2000;102(20 Suppl 4):IV14-IV23.

2. Hunter JJ, Chien KR. Signaling pathways for cardiac hypertrophy and failure. N Engl J Med 1999:341:1276-1283.

3. Ritter $\mathrm{O}$, Neyses $\mathrm{L}$. The molecular basis of myocardial hypertrophy and heart failure. Trends Mol Med 2003;9:313-321.

4. Baba HA, Takeda A, Nagano M. The influence of decreased leftventricular afterload on cardiac morphology in hypertrophied rat hearts. Int J Cardiol 1995;49:107-117.

5. Vetterlein F, Schrader C, Volkmann R, et al. Extent of damage in ischemic, nonreperfused, and reperfused myocardium of anesthetized rats. Am J Physiol Heart Circ Physiol 2003;285:H755-H765. E-pub 1 May 2003.

6. Hein S, Arnon E, Kostin S, et al. Progression from compensated hypertrophy to failure in the pressure-overloaded human heart: structural deterioration and compensatory mechanisms. Circulation 2003;107:984-991.
7. Schaper J, Froede R, Hein S, et al. Impairment of the myocardial ultrastructure and changes of the cytoskeleton in dilated cardiomyopathy. Circulation 1991;83:504-514.

8. Capasso JM, Palackal T, Olivetti G, et al. Left ventricular failure induced by longterm hypertension in rats. Circ Res 1990;66:1400-1412.

9. Weber KT. Targeting pathological remodeling: concepts of cardioprotection and reparation. Circulation 2000;102:1342-1345.

10. Frerichs FC, Dingemans KP, Brinkman K. Cardiomyopathy with mitochondrial damage associated with nucleoside reversetranscriptase inhibitors. N Engl J Med 2002;347:1895-1896.

11. Molnar M, Schröder JM. Pleomorphic mitochondrial and different filamentous inclusions in inflammatory myopathies associated with mtDNA deletions. Acta Neuropathol (Berl) 1998;96:41-51.

12. Li YY, McTiernan CF, Feldman AM. Interplay of matrix metalloproteinases, tissue inhibitors of metalloproteinases and their regulators in cardiac matrix remodeling. Cardiovasc Res 2000;46: 214-224.

13. Wang J, Xu N, Feng $X$, et al. Targeted disruption of Smad4 in cardiomyocytes results in cardiac hypertrophy and heart failure. Circ Res 2005;97:821-828. E-pub 8 September 2005.

14. Sivasubramanian N, Coker ML, Kurrelmeyer KM, et al. Left ventricular remodeling in transgenic mice with cardiac restricted overexpression of tumor necrosis factor. Circulation 2001;104:826-831.

15. Sarkar S, Leaman DW, Gupta S, et al. Cardiac expression of myotrophin triggers myocardial hypertrophy and heart failure in transgenic mice. J Biol Chem 2004;279:20422-20434. E-pub 16 February 2004.

16. Kostin S, Hein S, Arnon E, et al. The cytoskeleton and related protein in human heart. Heart Fail Rev 2000;5:271-280.

17. Pereira LM, Mandarim-De-Lacerda CA. Quantitative examination of the cardiac myocytes in hypertensive rats under chronic inhibition of nitric oxide synthesis. J Biomed Sci 1998;5:363-369.

18. Chomczynski P, Sacchi N. Single-step method of RNA isolation by acid guanidinium thiocyanate-phenol-chloroform extraction. Anal Biochem 1987;162:156-159.

19. Piper HM. Mitochondrial injury in the oxygen-depleted and reoxygenated myocardial cell. In: Piper HM (ed). Pathophysiology of Severe Ischemic Myocardial Injury. Kluwer Academic Publishers: Dordrecht, Boston, 1990, pp 91-113.

20. Dolman J, Godin DV. Myocardial ischaemic/reperfusion injury in the anaesthetized rabbit: comparative effects of halothane and isoflurane. Can Anaesth Soc J 1986;33:443-452.

21. Alexander $\mathrm{CM}$, Werb Z. Proteinases and extracellular matrix remodeling. Curr Opin Cell Biol 1989;1:974-982.

22. Brilla CG, Rupp H. Myocardial collagen matrix remodeling and congestive heart failure. Cardiologia 1994;39(12 Suppl 1):389-393.

23. Nikolova V, Leimena C, McMahon AC, et al. Defects in nuclear structure and function promote dilated cardiomyopathy in lamin A/C-deficient mice. J Clin Invest 2004;113:357-369.

24. Ozisik K, Yildirim E, Kaplan S, et al. Ultrastructural changes of rat cardiac myocytes in a time-dependent manner after traumatic brain injury. Am J Transplant 2004;4:900-904.

25. Tokuyasu KT, Dutton AH, Singer SJ. Immunoelectron microscopic studies of desmin (skeletin) localization and intermediate filament organization in chicken cardiac muscle. J Cell Biol 1983;96:1736-1742.

26. Hein S, Kostin S, Heling A, et al. The role of the cytoskeleton in heart failure. Cardiovasc Res 2000;45:273-278.

27. Heling A, Zimmermann R, Kostin $S$, et al. Increased expression of cytoskeletal, linkage, and extracellular proteins in failing human myocardium. Circ Res 2000;86:846-853. 